\title{
New lignin streams derived from heteropoly acids-enhanced neutral deep eutectic solvent fractionation: Towards structural elucidation and antioxidant performance
}

Zongwei Guo ${ }^{1,2}$, Deqiang $\mathrm{Li}^{1}$, Tingting You ${ }^{1}$, Xun Zhang ${ }^{1}$, Feng $\mathrm{Xu}^{1 *}$ Xueming, Zhang $^{1 *}$, Yiqin Yang ${ }^{2}$

${ }^{1}$ Beijing Key Laboratory of Lignocellulosic Chemistry, Beijing Forestry University, Beijing, 100083, China

${ }^{2}$ Jiangsu Provincial Key Laboratory of Pulp and Paper Science and Technology, Nanjing Forestry University, Nanjing, Jiangsu Province, 210037, China

*Corresponding authors

Tel: $+86-10-62337993 \quad$ Fax: $+86-10-62337993$

E-mail: xfx315@bjfu.edu.cn

xm_zhang@bjfu.edu.cn 
Number of pages: 6

Number of Tables: 2

Number of Figures: 1

Table S1. The solubility of lignin fractions.

Table S2. Assignments of ${ }^{13} \mathrm{C}-{ }^{1} \mathrm{H}$ correlated signals in the HSQC spectra of the lignin from bamboo.

Fig. S1. Photographs of lignin solubility in ethanol, tetrahydrofuran, dimethylacetamide solvents: (a) low solubility, (b) moderate solubility, (c) good solubility. 


\section{Table S1}

The solubility of lignin fractions.

\begin{tabular}{lllllll}
\hline \multirow{2}{*}{ Solvents } & \multicolumn{2}{l}{ HPA-DES lignin } & \multicolumn{2}{l}{ Alkali Lignin } & \multicolumn{2}{c}{ Lignosulphonate } \\
\cline { 2 - 6 } & $\begin{array}{l}\text { Solubility } \\
(\mathrm{mg} / \mathrm{mL})\end{array}$ & $\begin{array}{l}\text { Solubility } \\
(\mathrm{wt} \%)\end{array}$ & $\begin{array}{l}\text { Solubility } \\
(\mathrm{mg} / \mathrm{mL})\end{array}$ & $\begin{array}{l}\text { Solubility } \\
(\mathrm{wt} \%)\end{array}$ & $\begin{array}{l}\text { Solubility } \\
(\mathrm{mg} / \mathrm{mL})\end{array}$ & $\begin{array}{l}\text { Solubility } \\
(\mathrm{wt} \%)\end{array}$ \\
\hline Methanol & $<1$ & $<0.13$ & $<1$ & $<0.13$ & $<1$ & $<0.13$ \\
Ethanol & $<1$ & $<0.13$ & $<1$ & $<0.13$ & $<1$ & $<0.13$ \\
Acetone & $<1$ & $<0.13$ & $<1$ & $<0.13$ & $<1$ & $<0.13$ \\
Deionized water & $<1$ & $<0.10$ & $<3$ & $<3.00$ & $>100$ & $>10$ \\
Ethylacetate & $<1$ & $<0.11$ & $<1$ & $<0.11$ & $<1$ & $<0.11$ \\
Cyclohexane & $<1$ & $<0.13$ & $<1$ & $<0.13$ & $<1$ & $<0.13$ \\
Phenylcarbinol & $<1$ & $<0.10$ & $<1$ & $<0.10$ & $<1$ & $<0.10$ \\
Dichloroethane & $<1$ & $<0.08$ & $<1$ & $<0.08$ & $<1$ & $<0.08$ \\
1,4-dioxane & $<1$ & $<0.10$ & $<1$ & $<0.10$ & $<1$ & $<0.10$ \\
Tetrahydrofuran & 4 & 0.45 & 4 & 0.45 & 3 & 0.34 \\
Dimethyl sulfoxide & $>100$ & $>9.1$ & $>70$ & $>6.4$ & $>50$ & $>4.5$ \\
Dimethylacetamide & $>100$ & $>10.7$ & $>80$ & $>8.5$ & $>60$ & $>6.4$ \\
\hline
\end{tabular}




\section{Table S2}

Assignments of ${ }^{13} \mathrm{C}-{ }^{1} \mathrm{H}$ correlated signals in the HSQC spectra of the lignin from bamboo.

\begin{tabular}{|c|c|c|}
\hline Labels & $\delta_{\mathrm{C}} / \delta_{\mathrm{H}}(\mathrm{ppm})$ & Assignment \\
\hline $\mathrm{C}_{\beta}$ & $53.8 / 3.71$ & $\mathrm{C}_{\beta}-\mathrm{H}_{\beta}$ in phenylcomaran structures $(\mathrm{C})$ \\
\hline $\mathrm{B}_{\beta}$ & $53.8 / 3.07$ & $\mathrm{C}_{\beta}-\mathrm{H}_{\beta}$ in resinol substructures $(\mathrm{B})$ \\
\hline$-\mathrm{OCH}_{3}$ & $55.2 / 3.59$ & $\mathrm{C}-\mathrm{H}$ in methoxyls \\
\hline $\mathrm{A}_{\gamma}$ & $59.5 / 3.62$ & $\mathrm{C}_{\gamma}-\mathrm{H}_{\gamma}$ in $\beta-O-4$ substructures(A) \\
\hline $\mathrm{I}_{\gamma}$ & $62.3 / 4.09$ & $\begin{array}{l}\mathrm{C}_{\gamma}-\mathrm{H}_{\gamma} \text { in cinnamyl(sinapyl/coniferyl) alcohol end } \\
\text { groups(I) }\end{array}$ \\
\hline $\mathrm{X}_{5}$ & $62.8 / 3.25$ & $\mathrm{C}_{5}-\mathrm{H}_{5} \beta$-D-xylopyranoside substructures $(\mathrm{X})$ \\
\hline $\mathrm{B}_{\gamma}$ & $71.1 / 3.70$ & $\mathrm{C}_{\gamma}-\mathrm{H}_{\gamma}$ in resinol substructures(B) \\
\hline
\end{tabular}




\begin{tabular}{|c|c|c|}
\hline $\mathrm{A}_{\alpha}$ & $71.3 / 4.86$ & $\mathrm{C}_{\alpha}-\mathrm{H}_{\alpha}$ in $\beta-O-4$ substructures(A) \\
\hline $\mathrm{X}_{2}$ & $73.0 / 3.10$ & $\mathrm{C}_{2}-\mathrm{H}_{2} \beta$-D-xylopyranoside substructures $(\mathrm{X})$ \\
\hline $\mathrm{X}_{2}^{\prime}$ & $73.1 / 4.46$ & $\begin{array}{l}\mathrm{C}_{2}-\mathrm{H}_{2} \quad \text { in } \\
\text { substructures }(\mathrm{X})\end{array}$ \\
\hline $\mathrm{X}_{3}$ & $74.1 / 3.26$ & $\mathrm{C}_{3}-\mathrm{H}_{3} \beta$-D-xylopyranoside substructures $(\mathrm{X})$ \\
\hline $\mathrm{X}_{4}$ & $76.4 / 3.63$ & $\mathrm{C}_{4}-\mathrm{H}_{4} \beta$-D-xylopyranoside substructures $(\mathrm{X})$ \\
\hline $\mathrm{D}_{\alpha}^{\prime}$ & $79.4 / 4.01$ & $\mathrm{C}_{\alpha}-\mathrm{H}_{\alpha}$ in spirodienone substructures $\left(\mathrm{D}^{\prime}\right)$ \\
\hline $\mathrm{A}_{\beta}^{\prime}(\mathrm{G})$ & $80.8 / 4.61$ & $\mathrm{C}_{\beta}-\mathrm{H}_{\beta}$ in $\beta-O-4$ linked to a $\mathrm{G}(\mathrm{H})$ \\
\hline $\mathrm{B}_{\alpha}^{\prime}$ & $83.0 / 4.91$ & $\mathrm{C}_{\alpha}-\mathrm{H}_{\alpha}$ in $\beta-\beta\left(\mathrm{B}^{\prime}\right.$, tetrahydrofuran $)$ \\
\hline $\mathrm{A}_{\beta}(\mathrm{G})$ & $83.2 / 4.34$ & $\mathrm{C}_{\beta}-\mathrm{H}_{\beta}$ in $\beta-O-4$ linked to a $\mathrm{G}(\mathrm{H})$ \\
\hline $\mathrm{B}_{\alpha}$ & $84.8 / 4.62$ & $\mathrm{C}_{\alpha}-\mathrm{H}_{\alpha}$ in resinol substructures(B) \\
\hline$A_{\beta}(S)$ & $85.7 / 4.10$ & $\mathrm{C}_{\beta}-\mathrm{H}_{\beta}$ in $\beta-O-4$ linked to a $\mathrm{S}$ unit(A) \\
\hline $\mathrm{C}_{\alpha}$ & $87.5 / 5.48$ & $\mathrm{C}_{\alpha}-\mathrm{H}_{\alpha}$ in phenylcomaran structures $(\mathrm{C})$ \\
\hline$S_{2,6}$ & $103.7 / 6.59$ & $\mathrm{C}_{2,6}-\mathrm{H}_{2,6}$ in syringyl units $(\mathrm{S})$ \\
\hline $\mathrm{T}_{2,6}^{\prime}$ & $105.4 / 7.34$ & $\mathrm{C}_{2,6}-\mathrm{H}_{2,6}$ in tricin $(\mathrm{T})$ \\
\hline $\mathrm{G}_{2}$ & $111.1 / 6.94$ & $\mathrm{C}_{2}-\mathrm{H}_{2}$ in guaiacyl units $(\mathrm{G})$ \\
\hline $\mathrm{FA}_{2}$ & $111.3 / 7.32$ & $\mathrm{C}_{2}-\mathrm{H}_{2}$ in ferulate $(\mathrm{FA})$ \\
\hline $\mathrm{PCA}_{\beta} / \mathrm{FA}_{\beta}$ & $113.6 / 6.23$ & $\mathrm{C}_{\beta}-\mathrm{H}_{\beta}$ in $\mathrm{p}$-coumarate (PCA) and ferulate (FA) \\
\hline $\mathrm{G}_{5}$ & $114.5 / 6.70$ & $\mathrm{C}_{5}-\mathrm{H}_{5}$ in guaiacyl units $(\mathrm{G})$ \\
\hline $\mathrm{PCA}_{3,5}$ & $115.8 / 6.68$ & $\mathrm{C}_{3,5}-\mathrm{H}_{3,5}$ in p-coumarate (PCA) \\
\hline
\end{tabular}




\begin{tabular}{lll}
\hline $\mathrm{G}_{6}$ & $119.1 / 6.78$ & $\mathrm{C}_{6}-\mathrm{H}_{6}$ in guaiacyl units $(\mathrm{G})$ \\
$\mathrm{FA}_{6}$ & $122.5 / 7.13$ & $\mathrm{C}_{2,6}-\mathrm{H}_{2,6}$ in ferulate (FA) \\
$\mathrm{J}_{\beta}$ & $126.1 / 6.72$ & $\mathrm{C}_{\beta}-\mathrm{H}_{\beta}$ in cinnamyl aldehyde end-group (J) \\
$\mathrm{H}_{2,6}$ & $128.0 / 7.22$ & $\mathrm{C}_{2,6}-\mathrm{H}_{2,6}$ in $p$-hydroxyphenyl units $(\mathrm{H})$ \\
$\mathrm{PCA}_{2,6} / \mathrm{PCA}_{2,6}$ & $130.1 / 7.48$ & $\mathrm{C}_{2,6}-\mathrm{H}_{2,6}$ in $p$-coumarate (PCA) \\
$\mathrm{PCA}_{\alpha} / \mathrm{FA}_{\alpha}$ & $144.6 / 7.46$ & $\mathrm{C}_{\alpha}-\mathrm{H}_{\alpha}$ in p-coumarate (PCA) and ferulate (FA) \\
\hline
\end{tabular}


Fig. S1. Photographs of lignin solubility in ethanol, tetrahydrofuran, dimethylacetamide solvents: (a) low solubility, (b) moderate solubility, (c) good solubility.

a

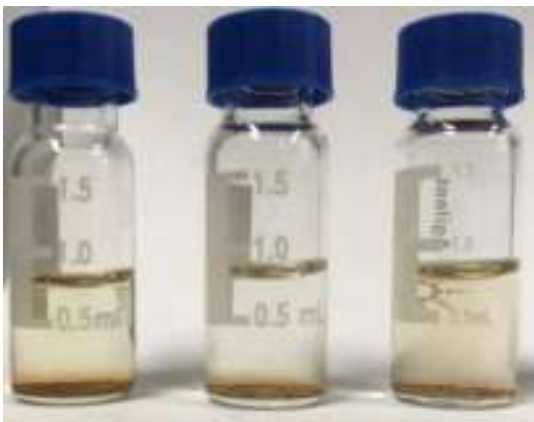

PTA PMA b

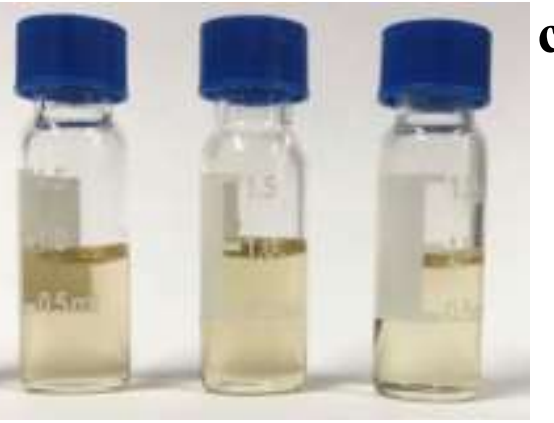

PTA

PMA STA

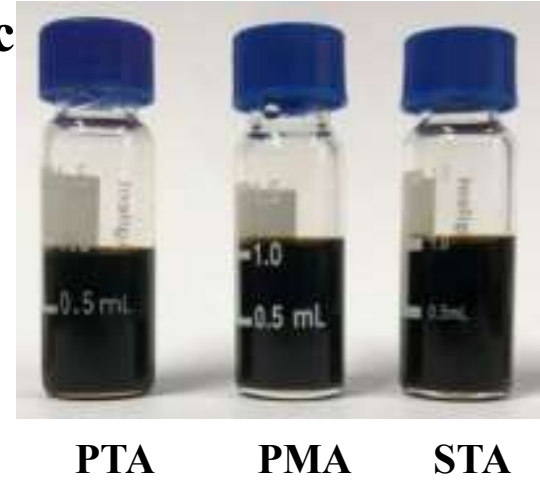

